\title{
The impact of beam angle configuration of intensity- modulated radiotherapy in the hepatocellular carcinoma
}

\author{
Sung Hoon Kim, MD1, Min Kyu Kang, MD', Ji Woon Yea, MD', Sung Kyu Kim, PhD', \\ Ji Hoon Choi, MD', Se An Oh, MS² \\ 'Department of Radiation Oncology, Yeungnam University College of Medicine, Daegu; \\ ${ }^{2}$ Department of Physics, Yeungnam University, Gyeongsan, Korea
}

Purpose: This treatment planning study was undertaken to evaluate the impact of beam angle configuration of intensitymodulated radiotherapy (IMRT) on the dose of the normal liver in hepatocellular carcinoma (HCC).

Materials and Methods: The computed tomography datasets of 25 patients treated with IMRT for HCC were selected. Two IMRT plans using five beams were made in each patient; beams with equidistance of $72^{\circ}$ (Plan I), and beams with a $30^{\circ}$ angle of separation entering the body near the tumor (Plan II). Both plans were generated using the same constraints in each patient. Conformity index $(\mathrm{Cl})$, homogeneity index (HI), gamma index, mean dose of the normal liver (Dmean_NL), Dmean_NL difference between the two plans, and percentage normal liver volumes receiving at least 10, 20, and $30 \mathrm{~Gy}(\mathrm{~V} 10, \mathrm{~V} 20$, and V30) were evaluated and compared.

Results: Dmean_NL, V10, and V20 were significantly better for Plan II. The Dmean_NL was significantly lower for peripheral $(p=$ $0.001)$ and central tumors $(p=0.034)$. Dmean_NL differences between the two plans increased in proportion to gross tumor volume to normal liver volume ratios $(p=0.002)$. $\mathrm{Cl}, \mathrm{HI}$, and gamma indices were not significantly different for the two plans.

Conclusion: The IMRT plan based on beams with narrow separations reduced the irradiated dose of the normal liver, which would allow radiation dose escalation for HCC.

Keywords: Hepatocellular carcinoma, Intensity modulated radiation therapy, Radiation therapy

\section{Introduction}

In the past, radiotherapy for the treatment of hepatocellular carcinoma (HCC) was limited to palliative treatment because the liver was considered radiosensitive and it was difficult to reduce radiation dose to the normal liver due to technical limitations. In recent years, high dose radical radiation therapy has been being attempted by using 3-dimensional conformal radiation therapy (3D-CRT) [1-4]. Furthermore, a doseresponse relationship exists in $\mathrm{HCC}$ and several studies have reported that radiation doses exceeding 45-50 Gy increase response rate [4-6]. However, even when 3D-CRT is used, it is difficult to prescribe high-dose radiation for large tumors due to the risk of radiation-induced liver disease $[7,8]$.

Intensity-modulated radiation therapy (IMRT) has been widely used to treat head and neck tumors and prostate cancer to reduce radiation-induced side effects. Recently, IMRT has been used to treat moving tumors, such as, those of lung cancer or HCC [9-12]. Mclntosh et al. [10] prescribed 50 Gy in 20 fractions to $20 \mathrm{HCC}$ patients using helical tomotherapy. Partial

Received 16 April 2012, Revised 26 May 2012, Accepted 13 June 2012.

Correspondence: Min Kyu Kang, MD, Department of Radiation Oncology, Yeungnam University College of Medicine, 170 Hyeonchung-ro, Nam-gu, Daegu 705-717, Korea. Tel: +82-53-620-3370, Fax: +82-53-624-3599, E-mail: mkkang@ynu.ac.kr

(c) This is an Open Access article distributed under the terms of the Creative Commons Attribution Non-Commercial License (http://creativecommons.org/ licenses/by-nc/3.0/) which permits unrestricted non-commercial use, distribution, and reproduction in any medium, provided the original work is properly cited. www.e-roj.org 
response or stable disease was achieved in 94\% and a median survival was 9.6 months. Jang et al. [11] irradiated 40-50 Gy in 10 fractions to intra- and extra-hepatic lesions simultaneously using helical tomotherapy and found that $45 \%$ of intrahepatic lesions achieved more than partial response. Kang et al. [12] prescribed 50.4 Gy in 28 fractions to 27 HCC patients by linear accelerator based IMRT, and 44\% achieved better than partial response.

In this study, we compared two radiotherapy plans based on five equidistance beams or 5 narrowly separated beams entering near tumors to determine the optimal beam angle required to reduce doses administered to the normal liver and to increase prescribed tumor doses.

\section{Materials and Methods}

Computed tomography (CT) datasets of 25 patients treated for HCC between April 2010 and February 2012 were used.

\section{Simulation and delineation}

During simulation, each of the 25 patients was placed supine with arms elevated above his/her head. CT was scanned with contrast enhancement at a slice thickness of $5 \mathrm{~mm}$. Gross tumor volume (GTV) and normal tissues were delineated using an Eclipse treatment planning system ver. 8.6.0 (Varian Medical System, Palo Alto, CA, USA). Planning target volume (PTV) was generated by adding $1-\mathrm{cm}$ radial margin and $1.5-\mathrm{cm}$ craniocaudal margin of the GTV. Total liver, normal liver (total liver minus GTV), kidneys, and spinal cord were delineated as organs at risk.
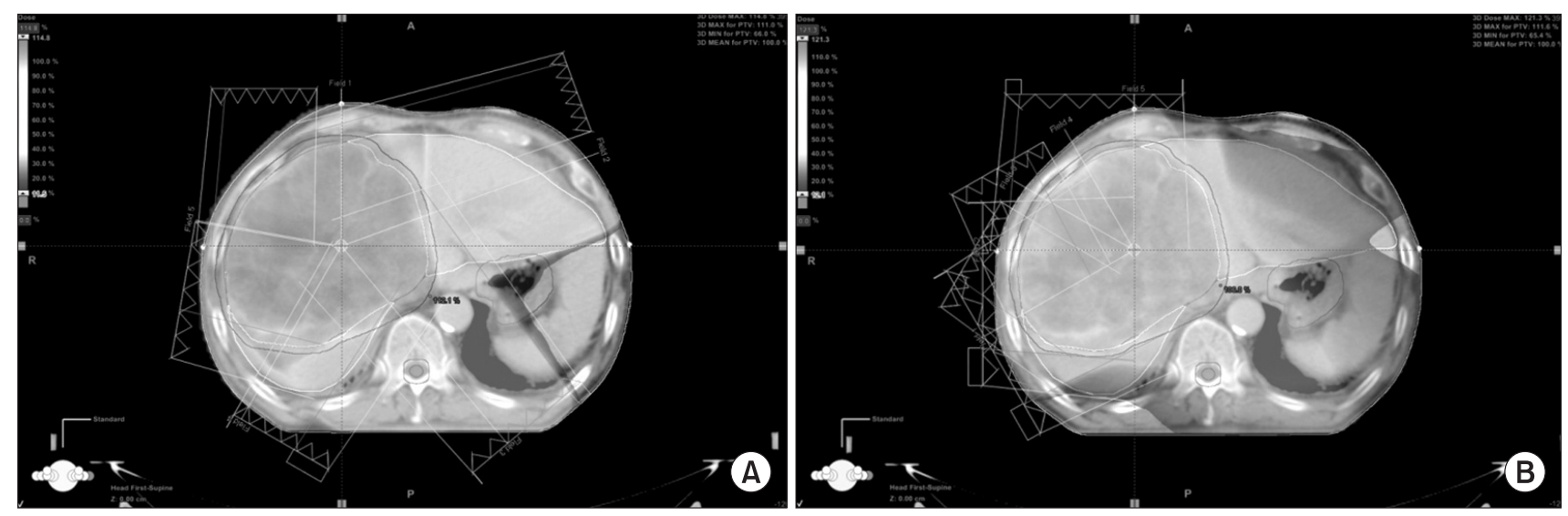

Fig. 1. Beam angle configuration. (A) Five beams, equally separated by $72^{\circ}$ (Plan I). (B) Beam alignment was weighted toward the tumor side of the patient's body (Plan II).

\section{Planning}

Five field step-and-shoot IMRT plans using 6-MV photon were generated using the Eclipse treatment planning system. First, Plan I was prepared using 5 equidistant beams set $72^{\circ}$ apart. While optimizing each plan, $40 \%$ of the normal liver volume was initially set to receive less than 2,000 cGy, and then volume or dose was modified as long as PTV was not compromised. The constraints used for optimization in all patients were that the percentage of normal liver volume receiving 1,200-2,000 cGy (median, 2,000 cGy) was 20-40\% (median, 30\%). The dose administered to $20 \%$ of the volume of each kidney was kept at less than 2,000 cGy and the maximum dose administered of the spinal cord was 3,000 cGy. A dose of 5,400 cGy in 30 fractions (daily $180 \mathrm{cGy}$ ) was prescribed to the mean dose of the PTV. If mean dose to the normal liver (Dmean_NL) exceeded 2,600 cGy, the prescribed dose was reduced to $5,040 \mathrm{cGy}$ in 28 and then to $4,500 \mathrm{cGy}$ in 25 fractions in stages to satisfy the criterion that Dmean_NL should be less than 2,600 cGy.

After making Plan I, Plan II was generated by modifying beam configuration to make the beams enter the body near the tumor with a $30^{\circ}$ angle of separation. All constraints of Plan I were used for the optimization of Plan || and the same total dose was prescribed in each patient (Fig. 1).

\section{Statistical analysis}

To evaluate the radiation dose administered to the normal liver by the two plans, Dmean_NL and percentage volumes of the normal liver receiving at least 10, 20, and 30 Gy (V10, V20, and V30) were compared for the two plans using the paired t-test or Wilcoxon signed rank test. In addition, subgroup analyses 
Table 1. Characteristics of target and normal-organ volumes

\begin{tabular}{lc}
\hline \multicolumn{1}{c}{ Characteristic } & Median (range) \\
\hline Total liver volume $(\mathrm{mL})$ & $1,583(636-4,119)$ \\
Normal liver volume $(\mathrm{mL})$ & $1,252(549-3,317)$ \\
Long diameter of GTV $(\mathrm{cm})$ & $9.5(2.6-21.0)$ \\
ESD of GTV $(\mathrm{cm})$ & $7.5(1.6-17.0)$ \\
GTV $(\mathrm{mL})$ & $218(2-2,557)$ \\
PTV $(\mathrm{mL})$ & $401(34-3,358)$ \\
GTV/normal liver volume & $0.19(0.01-1.78)$ \\
\hline
\end{tabular}

ESD, equivalent spherical diameter; GTV, gross tumor volume; PTV, planning target volume.

were performed with respect to tumor location and size. Tumors were divided into two groups according to location, that is, the central tumor group (around porta hepatis) and the peripheral tumor group (far from the porta hepatis and close to the chest wall). Differences between the two plans with respect to Dmean_NL, V10, V20, and V30 by tumor location were analyzed using the independent t-test. Pearson's correlation coefficient was used to compare the correlation between GTV to normal liver volume ratios and inter-plan differences in Dmean_NL, V10, V20, and V30 (Plan I minus Plan II).

The conformity index $(\mathrm{Cl})$ and homogeneity index $(\mathrm{HI})$ were used to evaluate the dose coverage of the PTV by each plan. $\mathrm{Cl}$ was originally proposed by Paddick [13] to evaluate tightness of fit between PTVs and isodose volumes in treatment plans as follows,

$\mathrm{Cl}=(\text { the volume of PTV covered by } 95 \% \text { isodose surface })^{2} /$ the volume of PTV $\times$ total volume receiving at least $95 \%$ of the prescribed dose

$\mathrm{HI}$ was defined as D5\%/D95\%, where D5\% and D95\% are the minimum doses delivered to 5\% and 95\% of the PTV. A higher $\mathrm{HI}$ value indicates greater PTV dose distribution inhomogeneity. Plan verification was performed using I'mRT MatriXX (IBA, Schwarzenbruck, Germany) by comparing gamma indices, that is, the percentage of detector points that passed the $3 \mathrm{~mm} / 3 \%$ gamma criterion. $\mathrm{Cl}, \mathrm{HI}$, and gamma indices were compared using the paired t-test or Wilcoxon signed rank test.

All analyses were performed using PASW ver. 18.0 (SPSS Inc., Chicago, IL, USA). A p-value less than 0.05 was considered significant.
Table 2. Comparisons of the dosimetric parameters of the two IMRT plans

\begin{tabular}{lccc}
\hline \multicolumn{1}{c}{ Variable } & Plan I & Plan II & p-value \\
\hline Dmean_NL (cGy) & $2,038 \pm 543$ & $1,893 \pm 492$ & $<0.001$ \\
V10 (\%) & $67.2 \pm 18.4$ & $59.8 \pm 17.0$ & $<0.001$ \\
V20 (\%) & $42.4 \pm 15.3$ & $36.8 \pm 13.8$ & 0.003 \\
V30 (\%) & $25.6 \pm 9.9$ & $25.3 \pm 9.0$ & 0.734 \\
\hline
\end{tabular}

Dmean_NL, mean dose of normal liver; IMRT, intensity-modulated radiotherapy.

a) Percentage volumes of normal liver receiving at least 10,20 , or $30 \mathrm{~Gy}$.

\section{Results}

\section{Tumor characteristics}

Median volumes of total and normal livers of the 25 patients were 1,583 $\mathrm{mL}$ (range, 636 to $4,119 \mathrm{~mL}$ ) and 1,410 $\mathrm{mL}$ (range, 549 to $3,317 \mathrm{~mL}$ ), respectively. Median GTV and PTV volumes were $218 \mathrm{~mL}$ (range, 2 to $2,557 \mathrm{~mL}$ ) and $401 \mathrm{~mL}$ (range, 34 to $3,358 \mathrm{~mL}$ ), respectively and the median GTV to normal liver volume ratio was 0.19 (range, 0.01 to 1.78 ) (Table 1).

There were 11 central and 14 peripheral tumors. Of the peripheral tumors, nine tumors were located across the right lobe antero-posteriorly, four tumors were located in the posterior aspect of the right lobe, and one tumor was located in the left lobe.

\section{Comparisons of dosimetric parameters}

Mean Dmean_NL of Plan II was 1,893 cGy (range, 886 to 2,575 cGy) and this was significantly lower than that of Plan I which was 2,038 cGy (range, 843 to 2,595 cGy; $p<0.001$ ), and mean Dmean_NL difference between the two plans was $120 \mathrm{cGy}$ (range, 56 to $574 \mathrm{cGy}$ ). V10 and V20 were significantly lower in Plan II, but $\mathrm{V} 30$ was no different (Table 2).

In terms of tumor locations, Dmean_NL and V10 and V20 of Plan II were significantly lower for peripheral tumors and Dmean_NL and V10 of Plan II were significantly lower for central tumors. Mean Dmean_NL differences were not dependent on tumor location (188 $\pm 162 \mathrm{cGy}$ for peripheral tumors vs. $89 \pm 120$ cGy for central tumors; $p=0.104$ ) (Table 3 ).

When Dmean_NL values were compared according to prescribed dose, the Dmean_NL of Plan II was significantly lower than that of Plan I for tumors administered a total dose of 5,400 cGy and the mean Dmean_NL difference was 134 $\pm 127 \mathrm{cGy}(\mathrm{p}<0.001)$. At a total dose of 5,040 cGy, Dmean 
Table 3. Dosimetric parameters of two IMRT plans according to tumor location

\begin{tabular}{lcccc}
\hline Location (no.) & Dmean_NL (cGy) & V10 (\%) & V20 (\%) & V30 (\%) \\
\hline Peripheral (14) & & & & \\
Plan I & $2,356 \pm 232$ & $76.5 \pm 12.2$ & $50.8 \pm 6.6$ & $31.5 \pm 5.9$ \\
Plan II & $2,168 \pm 259$ & $69.3 \pm 11.7$ & $42.5 \pm 8.5$ & $30.1 \pm 5.5$ \\
Difference & $188 \pm 162$ & $7.3 \pm 8.4$ & $8.3 \pm 8.5$ & $1.5 \pm 2.8$ \\
p-value & 0.001 & 0.006 & 0.003 & 0.075 \\
Central (11) & & & \\
Plan I & $1,632 \pm 560$ & $55.3 \pm 18.4$ & $31.6 \pm 16.7$ & $17.9 \pm 8.6$ \\
Plan II & $1,543 \pm 503$ & $47.8 \pm 15.2$ & $29.6 \pm 12.7$ & $19.3 \pm 9.2$ \\
Difference & $89 \pm 120$ & $7.5 \pm 7.0$ & $2.0 \pm 5.4$ & $-1.4 \pm 3.3$ \\
p-value & 0.034 & 0.005 & 0.239 & 0.196 \\
\hline
\end{tabular}

Dmean_NL, mean dose of normal liver; IMRT, intensity-modulated radiotherapy.

a) Percentage volumes of normal liver receiving at least 10, 20, or $30 \mathrm{~Gy}$.

Table 4. Tumor characteristics and mean normal liver doses according to prescribed dose

\begin{tabular}{cccccc}
\hline Prescribed dose (cGy) & Tumor location (no.) & Plan I (cGy) & Plan II (cGy) & Difference (cGy) & p-value \\
\hline 4,500 & Peripheral (5) & $2,425 \pm 151$ & $2,189 \pm 107$ & $236 \pm 229$ & 0.043 \\
5,040 & Peripheral (3) & $2,467 \pm 89$ & $2,417 \pm 143$ & $51 \pm 65$ & 0.285 \\
5,400 & Total (17) & $1,847 \pm 562$ & $1,713 \pm 495$ & $134 \pm 127$ & $<0.001$ \\
& Central (11) & $1,632 \pm 560$ & $1,543 \pm 503$ & $89 \pm 120$ & 0.034 \\
& Peripheral (6) & $2,244 \pm 302$ & $2,027 \pm 309$ & $216 \pm 100$ & 0.004 \\
\hline
\end{tabular}

$\mathrm{NL}$ of Plan II was lower by $51 \pm 65 \mathrm{cGy}$, but this was not significant ( $p=0.285)$. Furthermore, Dmean_NL of Plan II was significantly lower in tumors with a prescribed dose of 4,500 cGy and the mean Dmean_NL difference was $236 \pm 229$ $c G y(p=0.043)$. When tumors were divided by location, all tumors administered a total dose of 4,500 cGy or 5,040 cGy and 11 of 17 tumors administered a total dose of 5,400 cGy were peripheral. Dmean_NL of Plan II was significantly lower regardless of tumor location for tumors administered a total dose of 5,400 cGy (Table 4).

Dmean_NL difference in each patient increased significantly higher with GTV to normal liver volume ratio (Pearson's correlation coefficient $=0.596, p=0.002$ ). Furthermore, this correlation was significant for peripheral tumors (Pearson's correlation coefficient $=0.609, p=0.021$ ), but not for central tumors (Pearson's correlation coefficient $=0.288, p=0.390$ ) (Fig. 2A). According to prescription dose, this correlation was significant for those administered a total dose of 5,400 cGy (Pearson's correlation coefficient $=0.539, p=0.026$ ), but not for those administered 5,040 cGy (Pearson's correlation coefficient $=0.163, p=0.896$ ) or $4,500 \mathrm{cGy}$ (Pearson's correlation coefficient $=0.662, p=0.224$ ) (Fig. 2B).

\section{Indices of IMRT planning}

$\mathrm{Cl}, \mathrm{HI}$, and gamma indices were no different for the two plans (Table 5).

\section{Discussion and Conclusion}

IMRT using beams with a narrow separation entering the body near the tumor (Plan II) reduced the dose administered to the normal liver as compared with IMRT using five equidistant beams (Plan I). Furthermore, dose reduction to the normal liver was greater as the GTV to normal liver volume ratio increased.

Irradiated doses to the normal liver can be influenced by beam angle in IMRT plans. Hsieh et al. [14] found that a noncoplanar IMRT plan could reduce irradiated dose to the normal liver as compared with a coplanar IMRT plan in HCC patients. Srivastava et al. [15] reported IMRT with beam angles optimized using an algorithm was superior to manual beam angle selection in patients with head and neck and prostate cancer. In the present study, IMRT using beams with a narrow separation at entry near the tumor were found to reduce dose to the normal liver as comparison with 5 equidistant beams. However, $\mathrm{Cl}, \mathrm{HI}$, and gamman indics were no different for the two plans and showed that both plans were suitable for the 
A

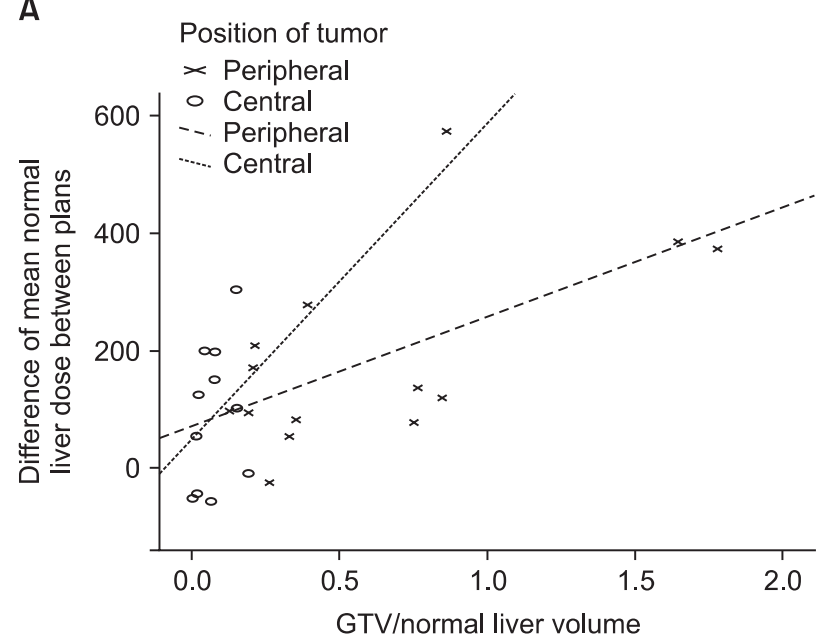

B

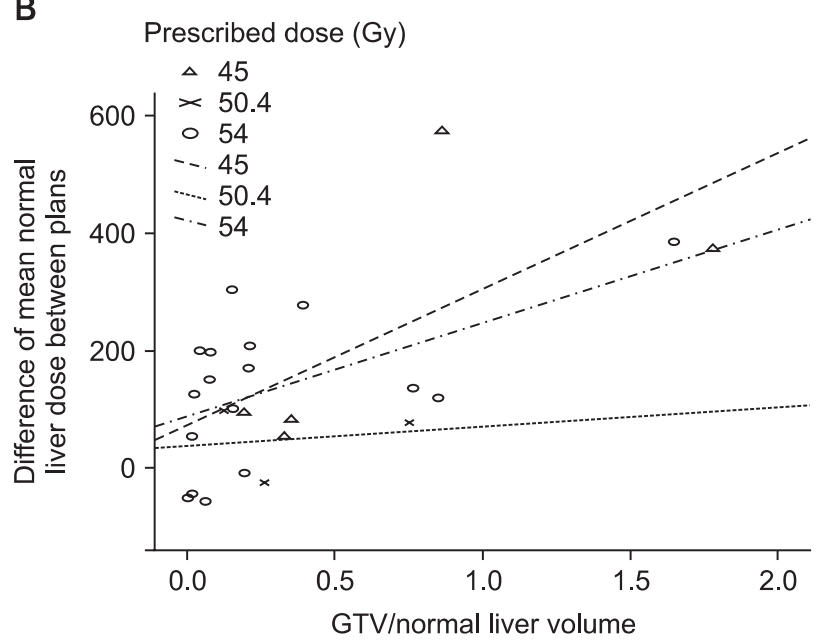

Fig. 2. Correlation between the differences between the mean normal liver doses of two plans and gross tumor volume (GTV) to normal liver volume ratios. (A) Correlation with respect to tumor location. Pearson's correlation coefficient was 0.288 for central tumors $(p=$ $0.390)$ and 0.609 for peripheral tumors $(p=0.021)$. (B) Correlation with respect to prescribed dose. Pearson's correlation coefficients were 0.662 at $45 \mathrm{~Gy}(\mathrm{p}=0.224), 0.163$ at $50.4 \mathrm{~Gy}(\mathrm{p}=0.896)$ and 0.539 at $54 \mathrm{~Gy}(\mathrm{p}=0.026)$.

clinical use.

When Dmean_NL differences were analyzed by tumor location, Dmean_NL was found to be lower for Plan II regardless of tumor location (Table 3). Although it was not significant, Dmean_NL difference was higher for peripheral tumors than central tumors ( $188 \pm 162$ cGy vs. $89 \pm 120$ cGy), probably because it is easier to decrease the paths of entering beams through the normal liver and to avoid beams passing through normal liver for peripheral tumors.

Dmean_NL difference significantly increased with GTV to normal liver volume ratio (Fig. 2). In terms of tumor location, this correlation was significant for peripheral tumors (Pearson's correlation coefficient $=0.609$ ), but not for central tumors. In terms of prescribed doses, this correlation was significant for those prescribed 5,400 cGy (Pearson's correlation coefficient = 0.539). Although Pearson's correlation coefficient was as high as 0.662 in those prescribed 4,500 cGy, this correlation was not significant due to a small number of patients. These results suggest that IMRT plans based on narrowly separated beams could reduce the irradiated dose to the normal liver, especially for large peripherally located tumors.

Dmean_NL differences between the two plans were significantly different between patients prescribed 5,400 cGy or 4,500 cGy (Table 4). In 5 tumors whose prescribed dose was reduced to 4,500 cGy, Dmean_NL was reduced by an average 236 cGy by changing beam configuration, which means total dose could have been increased to this extent.

This study has some limitations that warrant consideration.
Table 5. Comparison of the indices between the two IMRT plans

\begin{tabular}{lccc}
\hline \multicolumn{1}{c}{ Index } & Plan I & Plan II & p-value \\
\hline Conformity index & $0.84 \pm 0.06$ & $0.83 \pm 0.06$ & 0.183 \\
Homogeneity index & $0.94 \pm 0.01$ & $0.94 \pm 0.01$ & 0.199 \\
Gamma index (\%) & $99.4 \pm 0.9$ & $99.2 \pm 1.5$ & 0.331 \\
\hline
\end{tabular}

IMRT, intensity-modulated radiotherapy.

Tumors were heterogeneous in terms of the size and location and prescribed dose. Especially for central tumors, since they are usually recruited for radiotherapy earlier due to portal vein thrombosis hindering transarterial chemoembolization or the location preventing radiofrequency ablation, they tended to be smaller than peripheral tumors. For these reasons, only central tumors were prescribed a dose of 5,400 cGy in this study and Plan II was also found to be beneficial for the treatment of these tumors, although the Dmean_NL difference was small (Table 4).

In conclusion, IMRT plans based on beams with narrow separation reduce irradiated dose to the normal liver. By using this technique, it may be possible to increase tumor doses and improve treatment results in HCC.

\section{Conflict of Interest}

No potential conflict of interest relevant to this article was reported. 


\section{Acknowledgments}

This work was supported by a grant from the Chunma Medical Research Foundation, Korea (2011).

\section{References}

1. Park W, Lim DH, Paik SW, et al. Local radiotherapy for patients with unresectable hepatocellular carcinoma. Int J Radiat Oncol Biol Phys 2005;61:1143-50.

2. Krishnan S, Dawson LA, Seong J, et al. Radiotherapy for hepatocellular carcinoma: an overview. Ann Surg Oncol 2008; 15:1015-24.

3. Dawson LA. The evolving role of radiation therapy in hepatocellular carcinoma. Cancer Radiother 2008;12:96-101.

4. Ben-Josef $E$, Normolle $D$, Ensminger WD, et al. Phase II trial of high-dose conformal radiation therapy with concurrent hepatic artery floxuridine for unresectable intrahepatic malignancies. J Clin Oncol 2005;23:8739-47.

5. Seong J, Park HC, Han KH, Chon CY. Clinical results and prognostic factors in radiotherapy for unresectable hepatocellular carcinoma: a retrospective study of 158 patients. Int J Radiat Oncol Biol Phys 2003;55:329-36.

6. Liu MT, Li SH, Chu TC, et al. Three-dimensional conformal radiation therapy for unresectable hepatocellular carcinoma patients who had failed with or were unsuited for transcatheter arterial chemoembolization. Jpn J Clin Oncol 2004;34: 532-9.

7. Seong J. Challenge and hope in radiotherapy of hepatocellular carcinoma. Yonsei Med J 2009;50:601-12.

8. Pan CC, Kavanagh BD, Dawson LA, et al. Radiation-associated liver injury. Int J Radiat Oncol Biol Phys 2010;76(3 Suppl):S94100.

9. Jiang ZQ, Yang K, Komaki R, et al. Long-term clinical outcome of intensity-modulated radiotherapy for inoperable non-small cell lung cancer: the MD Anderson experience. Int J Radiat Oncol Biol Phys 2012;83:332-9.

10. McIntosh A, Hagspiel KD, Al-Osaimi AM, et al. Accelerated treatment using intensity-modulated radiation therapy plus concurrent capecitabine for unresectable hepatocellular carcinoma. Cancer 2009;115:5117-25.

11. Jang JW, Kay CS, You CR, et al. Simultaneous multitarget irradiation using helical tomotherapy for advanced hepatocellular carcinoma with multiple extrahepatic metastases. Int J Radiat Oncol Biol Phys 2009;74:412-8.

12. Kang MK, Kim MS, Kim SK, et al. High-dose radiotherapy with intensity-modulated radiation therapy for advanced hepatocellular carcinoma. Tumori 2011:97:724-31.

13. Paddick I. A simple scoring ratio to index the conformity of radiosurgical treatment plans: technical note. J Neurosurg 2000;93 Suppl 3:219-22.

14. Hsieh CH, Liu CY, Shueng PW, et al. Comparison of coplanar and noncoplanar intensity-modulated radiation therapy and helical tomotherapy for hepatocellular carcinoma. Radiat Oncol 2010;5:40.

15. Srivastava SP, Das IJ, Kumar A, Johnstone PA. Dosimetric comparison of manual and beam angle optimization of gantry angles in IMRT. Med Dosim 2011;36:313-6. 